Disclosure(s) Martin Feickert and Bjoern B. Burckhardt declare that there is no conflict of interest. This research did not receive any specific grant from funding agencies in the public, commercial, or not-for-profit sectors.

\section{P36 WHAT CAN BE LEARNT FROM SERIOUS DRUG RELATED MEDICAL ERRORS IN PEDIATRIC PATIENTS IN GOTHENBURG 2015-2017?}

S Fjeldseth*. Drottning Silvias Barn Sjukhus (DSBUS), Barnmedicin, Sahlgrenska University Hospital, Göteborg, Sweden

\subsection{6/archdischild-2019-esdppp.74}

Background 2017 a rise in serious drug related medical errors in pediatric care at Sahlgrenska University hospital was discovered. 2018 a pediatric Clinical Decision Support System (CDSS) called ePed with weight based dose calculation, dosage control and easy access to pediatric drug information was integrated with the Electronic Medical Record.

Aim Analyze causes and underlying factors to serious drug related medical errors, labeled Lex Maria, in children and further evaluate if these errors could be prevented within the framework of ePed.

Method 28 Lex Maria cases in children $\leq 16$ years were registered during 2015-2017 at Sahlgrenska University hospital, Gothenburg. These were analyzed and summarized according to type of error, underlying cause and when during the drug managing process the error occurred. Finally, comparison between years was made.

Results 2015 and 2016 five Lex Maria cases occurred respectively. 2017 the number increased to 18 cases. Most commonly the patients received an incorrect dose. Errors divide equally between prescription, preparation and administration. Drugs requiring dilution were of certain risk especially when diluted in several steps. Midazolam was the most frequently involved drug. In 2017 especially one ward stands out in numbers and was responsible for 10 of the 18 cases.

Conclusions ePed will help to avoid human calculation errors in prescription and provides easy access to pediatric drug information facilitating drug preparation and administration. Nurses are particularly exposed to medication incidents since they usually are the last link to the patient and need to be their own control. High-risk medicines need to be identified and made safe. A complex interplay between individual/human and systemic factors contribute to errors. However, to reduce serious drug related errors a CDSS is of great value but adequate education for medical staff, both pharmacologically and about the system is vital. Future follow up will be needed.

Disclosure(s) Nothing to disclose

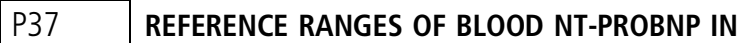 PAEDIATRIC HEART FAILURE AND HEALTHY CONTROLS: COMPILATION OF LITERATURE DATA}

T Gangnus*, FK Suessenbach, N Makowski, I Burdman, S Laeer, BB Burckhardt. Institute of Clinical Pharmacy, Heinrich Heine University, Dusseldorf, Germany

10.1136/archdischild-2019-esdppp.75

Background N-terminal pro-brain natriuretic peptide (NTproBNP) is a valuable biomarker for diagnosis and prognosis of heart failure in adults, included into the European Society Guidelines for heart failure (2016). ${ }^{1}$ It is also considered as a diagnostic and follow-up marker in paediatric heart failure. The aetiology of paediatric heart failure is heterogeneous and maturation of the cardiac and neurohumoral system influences NT-proBNP levels. Since substantial information is mandatory to enable a long-term follow-up of children with heart failure, the aim was to collect published paediatric NT-proBNP data. Methods In January 2019, a literature search using PubMed was performed comprising the following keywords: NTproBNP, heart failure/dilated cardiomyopathy/congenital heart defect/congenital heart disease/healthy and child/neonate/toddler/infant/paediatric. Eligible publications had to determine levels of NT-proBNP in plasma or serum in paediatric heart failure or healthy children (0-18 years) with the Roche NTproBNP-immunoassay.

Results The search resulted in 343 records, of which 95 measured NT-proBNP in paediatric controls or heart failure. Of them, 48 studies were excluded due to the use of other immunoassays. Following, 47 studies were included into the analysis of which 27 reported NT-proBNP levels in 3435 healthy children and 38 NT-proBNP concentrations in 1885 children with heart failure. The age range of reported levels comprised the day of birth up to 18 years in both groups. The data set revealed that younger children have higher NTproBNP values than older children and that heart failure patients had increased NT-proBNP levels compared to healthy controls which are also dependent on the severity of disease.

Conclusion The literature search and analysis confirmed that NT-proBNP is an important marker for the detection of heart failure and classification of disease severity in children. Thus, the compiled data set forms a solid data basis for long-term follow-up of a paediatric patient population with heart failure.

\section{REFERENCES}

1. Ponikowski P, Voors AA, Anker SD, et al. 2016 ESC Guidelines for the diagnosis and treatment of acute and chronic heart failure: The task force for the diagnosis and treatment of acute and chronic heart failure of the european society of cardiology (ESC)developed with the special contribution of the heart failure association (HFA) of the esc. Eur Heart J 2016;37(27):2129-200.

Disclosure(s) Tanja Gangnus, Fabian K. Suessenbach, Nina Makowski, Ilja Burdman, Stephanie Laeer, Björn B. Burckhardt declare that there is no conflict of interest. The research leading to these results has received funding from the European Union Seventh Framework programme (FP/2007-2013) under grant agreement $n^{\circ} 602295$ (LENA).

\section{P38 IDENTIFICATION OF NIFURTIMOX METABOLITES IN URINE OF PEDIATRIC CHAGAS DISEASE PATIENTS BY UHPLC-MS/MS}

CA Pérez Montilla, S Moroni, N González, G Moscatelli, JM Altcheh, F García Bournissen*. Multidisciplinary Institute for Research in Pediatric Pathologies (IMIPP-CONICET), Buenos Aires Children's Hospital 'Dr. Ricardo Gutiérrez', Ciudad Autónoma de Buenos Aires, Argentina

\subsection{6/archdischild-2019-esdppp.76}

Background Nifurtimox (NFX) is one of the only 2 available drugs for the treatment of Chagas disease, a parasitic disease endemic to Latin America. In spite of widespread use of this medication, little is known regarding its metabolism, particularly in children. 